Research Paper

\title{
The Basic Social Medical Insurance Is Associated with Clinical Outcomes in the Patients with ST-elevation Myocardial Infarction: A Retrospective Study from Shanghai, China
}

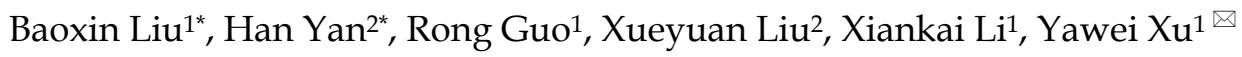 \\ 1. Department of Cardiology, Shanghai Tenth People's Hospital, Tongii University School of Medicine, Shanghai, 200072, China \\ 2. Department of Neurology, Shanghai Tenth People's Hospital, Tongji University School of Medicine, Shanghai, 200072, China
}

* These authors contributed equally to this work and should be considered co-first authors.

$\triangle$ Corresponding author: Professor Yawei Xu, MD, PhD. Email: xuyaweicn@sina.com Address: Department of Cardiology, Shanghai Tenth People's Hospital, Tongji University School of Medicine, 301 Yan Chang Zhong Road, Shanghai, 200072, China. Tel: +86-21-66306920 Fax: $+86-21-66307239$.

(c) Ivyspring International Publisher. This is an open-access article distributed under the terms of the Creative Commons License (http://creativecommons.org/ licenses/by-nc-nd/3.0/). Reproduction is permitted for personal, noncommercial use, provided that the article is in whole, unmodified, and properly cited.

Received: 2014.02.19; Accepted: 2014.05.29; Published: 20I4.06.22

\begin{abstract}
Objective: Several social economic factors play important roles in treatments of ST-elevation myocardial infarction (STEMI) and finally influence the clinical outcomes. The basic social medical insurance (BSMI) is an important economic factor in China's medical system. However, the impact of BSMI on clinical outcomes in STEMI patients has not been explored yet. The aim of this study is to investigate whether BSMI is a predictor of clinical outcomes in the patients with STEMI in Shanghai, China.

Material and methods: In this retrospective study, 68I STEMI patients from different areas in Shanghai were classified into four groups: new rural cooperative medical scheme (NCMS) group, urban resident basic medical insurance scheme (URBMI) group, urban employee basic medical insurance scheme (UEBMI) group and UNINSURED group, major adverse events (cardiac death, nonfatal reinfarction, clinically driven target lesion revascularization/target vessel revascularization, stroke, heart failure) were regarded as study endpoints to determine whether BSMI was a prognostic factor.

Results: During a mean follow-up of 36 months, the incidence of major adverse events was significantly higher in NCMS patients (64; 38.8\%) compared with the other groups: URBMI (47; 24.6\%); UEBMI (28; I5.6\%); UNISURED (40; 27.6\%). Similarly, cardiac mortality was also higher in NCMS group (19; I I.5\%). A Kaplan-Meier survival analysis revealed significantly lower event-free survival rate for major adverse events $(p<0.00 \mathrm{I})$ and cardiac mortality $(p=0.0 \mathrm{I})$ in NCMS group. Multivariate Cox regression analysis revealed that BSMI was an important prognostic factor in STEMI patients.

Conclusion: These results demonstrate that BSMI is closely associated with the major adverse events-free survival rate at 36-month follow-up in the STEMI patients under the current policies in Shanghai, China.
\end{abstract}

Key words: ST-elevation myocardial infarction; Basic social medical insurance; Commercial health insurance; Health inequality; Survival.

\section{Introduction}

ST-elevation myocardial infarction (STEMI) is a serious clinical type of coronary artery disease (CAD), which is characterized by ST-segment elevation in relevant leads on electrocardiogram (ECG). In China,
STEMI has now been a major public health problem for its high morbidity and mortality following the formation of the aging society in recent years [1].

The pathogenesis of STEMI is thrombosis and 
occlusion of coronary arteries, which results from the formation and rupture of vulnerable coronary atherosclerotic plaques. Therefore, the most effective treatment of STEMI is prompt and complete restoration of flow in the infarct artery. This can be achieved by reperfusion therapy such as thrombolysis, percutaneous transluminal coronary angioplasty (PTCA)/ percutaneous coronary intervention (PCI), or coronary artery bypass grafting (CABG). However, some studies [2,3] have found that, a lot of STEMI patients did not receive thrombolytic therapy or undergo PCI and even did not call for an ambulance after onset of STEMI. For the STEMI patients who received reperfusion therapy in Beijing, the median door-to-needle time for thrombolysis was 83 minutes and door-to-balloon time for primary PCI was 132 minutes [4], which is far from the 2013 American College of Cardiology Foundation/American Heart Association Guideline for the management of patients with STEMI recommended $<30 \mathrm{~min}$ for door-to-needle time for thrombolysis and $<90 \mathrm{~min}$ for door-to-balloon time for primary PCI [1]. Thus the clinical outcomes may be significantly affected. This delay in therapy for STEMI may be caused by multiple and complex factors.

Recently, socioeconomic status as a critical factor in the outcomes of diseases has been reported by $\mathrm{Wu}$ et al. [5]. Economic factors could also have significant effects on the therapy and clinical outcomes of STEMI. The medical cost, an important economic factor, had been rising significantly in China [6]. Reduction of medical cost could promote patients' life satisfaction which is potentially helpful to the therapy of STEMI [7]. Since disparities in medical cost are associated with different social medical insurance schemes [8], we hypothesize that the medical insurance is a crucial economic factor in the outcomes of patients with STEMI.

The medical insurance in China is composed of basic social medical insurance (BSMI) and commercial health insurance. BSMI is the countrywide government system that serves as the primary third-party payer and the backbone for healthcare financing [6]. It consists of three schemes including the new rural cooperative medical scheme (NCMS) for the rural population; the urban employee basic medical insurance scheme (UEBMI) for the urban employed and urban resident basic medical insurance scheme (URBMI) for urban residents. NCMS is characterized by its being government-funded on a voluntary basis. It was designed as a mutual help and risk-pooling health protection system. UEBMI requires the enrollment of all urban employees. Employers and employees share the responsibility of paying premium contributions. URBMI mainly covers urban residents who are not officially employed such as elderly, students or children. The pooling fund is mainly used for the medical cost of inpatient treatments and serious diseases in outpatient clinics; the medical cost of usual outpatient care was not covered. These three schemes function independently and vary across regions within China, with significant differences in the growth and policies of each particular scheme. Combined together, China's basic social medical insurance is providing coverage for more than 1.25 billion people [9]. Commercial health insurance in China serves as a supplement to social medical insurance providing substantial reimbursement with high premiums. According to the China Insurance Regulatory Commission, commercial health insurance accounted for only $6.03 \%$ of all medical insurance premiums and $5.72 \%$ of all insurance reimbursements in 2008 [9].

In Shanghai, the out-of-pocket medical cost (after insurance reimbursement) of inpatient treatments for STEMI patients depends on different insurance schemes. According to the policies issued by China Shanghai government, NCMS patients' reimbursement percentage differs from $50 \%$ to $70 \%$ in various hospitals. All NCMS patients with STEMI should pay $50 \%$ in tertiary hospitals. UEBMI only provides reimbursement for the participants whose gross medical cost (before insurance reimbursement) were more than the minimal cost, otherwise they will pay all the gross medical cost. The minimal cost depends on when they retire $(55-65$ years: $1.2 \mathrm{~K}$; $>65$ years: $0.7 \mathrm{~K}$, all in RMB). For the UEBMI patients who meet the condition, the reimbursement is $85 \%$ (retired at 55-65 years) or $92 \%$ (retired at $>65$ years) of the part more than the minimal cost. The reimbursement percentage in URBMI patients is $50 \%$ to $70 \%$, which varies with age ( $<60$ years including students and children: $50 \%$; $60-70$ years: $60 \%$; $>70$ years: $70 \%$ ). Therefore, the existing BSMI may result in different out-of-pocket medical cost which could influence the therapeutic compliance of the patients, and the clinical outcomes were thus different. Moreover, the gap between the government's capacity to pay and the rising pressures from healthcare demands might become overwhelming [9]. To date, the influence of BSMI on clinical outcomes in the STEMI patients has received little attention. The purpose of this study is to investigate the relationship between BSMI and clinical outcomes in the patients with STEMI.

\section{Material and Methods}

\section{Data source and patient population}

From February 2010 to January 2013, a total of 681 patients diagnosed as STEMI were enrolled in this retrospective study. Data on cardiac biomarker, 
echocardiographic and coronary angiography (CAG) findings were collected. The demographics, including risk factors for CAD (age, gender, hypertension, smoking, diabetes mellitus, hyperlipidemia) and BSMI-related economic factors (gross medical cost, out-of-pocket cost, household income, commercial health insurance) were documented. Medications including antiplatelet drugs, statins, $\beta$-blockers, angiotensin converting enzyme inhibitors (ACEI), angiotensin II receptor antagonists (ARB), calcium channel blocker (CCB), diuretics, and digitalis were also noted. According to the prescribed medication recorded in the study, the dual anti-platelet therapy (DAPT) adherence was evaluated. DAPT adherence referred to the use of scheduled clopidogrel plus aspirin medication for at least 1 year after discharge.

Patients who had a confirmed diagnosis of STEMI were eligible to participate in this study if 1) the age ranges from 18 to 80 years; 2) the patients were able to understand the study content and provide consent; 3) the patients were willing to accept the necessary follow-up, therapy and laboratory examination. The exclusion criteria included 1) patients with non-ST elevation myocardial infarction; 2) the patients with renal failure, severe liver disease; 3) patients with a life expectancy of $\leq 12$ months; 4) pregnant and lactating women; 5) the patients who were unable to understand the study content, or provide consent; 6) the patients who participate in the other study program in the meantime.

The study was approved by the institutional ethics committee of Shanghai Tenth People's Hospital, and all enrolled patients gave informed written consent to the study.

\section{Follow-up and endpoints}

All patients were followed up at 1, 3, 6, 12, 18, 24, 30 and 36 months after discharge. The medical history was taken, as well as prescribed medication was evaluated by medical review, and relevant examinations were performed if necessary. Patients were also asked carefully at each follow-up regarding the presence or absence of relevant symptoms. The length of follow-up was measured from the initial cardiac event.

Clinical follow-up data were gathered by reviewing outpatient records. Study endpoint is the 3 -year major adverse events which consisted of: 1) cardiac death; 2) a nonfatal reinfarction; 3) clinically driven target lesion revascularization (TLR) or target vessel revascularization (TVR); 4) stroke; 5) heart failure requiring unplanned office visits, emergency room visits, or hospitalization. The cardiac mortality was also analyzed separately in the study.

\section{Definitions}

Hypertension was defined as systolic blood pressure/diastolic blood pressure $\geq 140 / 90 \mathrm{mmHg}$ in the supine position, or the use of antihypertensive medication. Diabetes mellitus was identified by a fasting plasma glucose $\geq 7.0 \mathrm{mmol} / \mathrm{L}$, or random plasma glucose $\geq 11.1 \mathrm{mmol} / \mathrm{L}$, or if patients received therapy using insulin or oral medications for diabetes. Hyperlipidemia were defined as the presence of total cholesterol $\geq 5.7 \mathrm{mmol} / \mathrm{L}$, or $\mathrm{LDL} \geq 3.6 \mathrm{mmol} / \mathrm{L}$, or HDL $<1.04 \mathrm{mmol} / \mathrm{L}$, or current lipid-lowering medication use. Tobacco use, either at time of the interview or in the past, was recorded. The Killip classification was used to provide a clinical estimate of cardiac function [10]: Stage I: No heart failure - no clinical signs of cardiac decompensation; Stage II: Heart failure - diagnostic criteria including rales, S3 gallop and pulmonary venous hypertension, pulmonary congestion and wet rales in the lower half of the lung fields; Stage III: Severe heart failure - Frank pulmonary oedema with rales throughout the lung fields; Stage IV: Cardiogenic shock - signs including hypotension (systolic blood pressure $<90 \mathrm{mmHg}$ ), and evidence of peripheral vasoconstriction such as oliguria, cyanosis and diaphoresis. Cardiac death was defined as any death caused by a primary cardiovascular problem, including sudden and nonsudden cardiac death [11]. Clinically driven TLR was considered to be ischemia-driven if the target lesion diameter stenosis was $\geq 50 \%$ by quantitative analysis with either electrocardiographic changes at rest or a positive functional study in the distribution of the target lesion, or $\geq 70 \%$ with recurrent symptoms only. The target lesion was defined as the coronary segment containing the stent plus a 5-mm proximal or distal margin [12]. TVR was defined as the need for either PCI or CABG of the initial vessel intervened upon and excludes subsequent revascularization of a newly diseased or previously diseased vessel(s) [13]. The diagnosis of STEMI was according to World Health Organization definition of myocardial infarction (2008-09 revision) [14]. Incident myocardial infarction is defined as the person's first myocardial infarction. When features of myocardial infarction occur after 28 days of an incident event, the event is considered to be a recurrent myocardial infarction. If features of myocardial infarction occur in the first 28 days after an incident event, it is not counted as a new event for epidemiological purposes [15]. The diagnosis of stroke was according to 2013 American Heart Association/American Stroke Association Guidelines and 2013 Updated Definition [16, 17]. The diagnosis of heart failure was according to the 2013 American College of Cardiology Foundation/American Heart 
Association Guideline for the management of heart failure [18].

\section{Statistical analysis}

Results were expressed as mean \pm standard deviation (SD) for continuous variables and frequencies for categorical variables. Differences between groups were examined by nonparametric test and chi-square test for continuous and categorical variables respectively. The effect of different medical insurance schemes on clinical outcomes was assessed with the use of a multivariate Cox proportional hazards model. Other variables that were significantly associated with outcomes were entered into the model in a stepwise procedure. Event-free survival curves were constructed using the Kaplan-Meier method and compared using the log-rank test. An alpha value of 0.05 , corresponding to a $p$ value $<0.05$, served as criterion for establishing statistical significance. The 95\% confidence intervals of the hazard ratio were reported for all of the significant risk factors. Analysis was performed using SPSS for Windows (SPSS Inc., Version 16.0, Chicago, Illinois).

\section{Results}

\section{Baseline characteristics}

Among the 1341 STEMI patients inquired initially, 336 were not eligible and 173 not interested in the study. Over a period of follow-up, 90 patients were no longer interested and 61 patients without response to follow-up calls. The mean age of finally enrolled 681 patients is $62.8 \pm 12.6$. Female patients enrolled were 329 , corresponding to 352 male patients were enrolled in this study. According to different BSMI, 165 patients were included in NCMS group, 191 patients were in the URBMI group and 180 patients were in the UEBMI group. The remaining 145 patients without medical insurance were assigned to UNINSURED group (Figure 1). The demographic comparison and drug therapy of the enrolled patients were summarized in Table 1. The incidence of CAD risk factors such as hypertension, hyperlipidemia, and tobacco use were not significantly different in four groups. Moreover, the cardiac function classification and the house localization of the participants showed no significant difference. However, age of the enrolled patients showed significant difference $(p<0.001)$. The patients in the UEBMI and UNINSURED groups seem to be younger than the patients in the remaining two groups $(p<0.001)$. According to the 2013 American College of Cardiology Foundation/American Heart Association Guideline for the management of patients with STEMI, old age ( $\geq 65$ years) is a major risk factor of CAD [1]. The proportion of patients $\geq 65$ years with a major adverse cardiac event was $29.7 \%$ and $18.8 \%$ in NCMS group and URBMI group, respectively (Table 2; Figure 2). However, this proportion was significantly lower in UEBMI and UNINSURED groups (3.3\% and $6.2 \%$, respectively). The majority of patients with a major adverse event in UEBMI and UNINSURED groups were $<65$ years $(12.2 \%$ and $21.4 \%$, respectively) (Table 2; Figure 2). Since aspirin and clopidogrel utilization $(\mathrm{n} ; \%)$ were lower in NCMS group [(141; 85.5\%) and $(117 ; 70.9 \%)$, respectively] ( $p<0.001$ and $p=0.001$, respectively), the NCMS patients were thus less adherent to DAPT compared with other three groups $(117 ; 70.9 \%, \mathrm{p}<0.001)$. 
Table I. Baseline characteristics of enrolled patients.

\begin{tabular}{|c|c|c|c|c|c|}
\hline & NCMS group (n=165) & URBMI group $(n=191)$ & UEBMI group (n=180) & $\begin{array}{l}\text { UNINSURED group } \\
(\mathrm{n}=145)\end{array}$ & p value \\
\hline \multicolumn{6}{|l|}{ Characteristics } \\
\hline Age (years) & $68.7 \pm 10.2$ & $69.0 \pm 11.1$ & $54.4 \pm 7.1$ & $58.5 \pm 14.3$ & $<0.001$ \\
\hline Gender (M/F) & $83 / 82$ & $88 / 103$ & $103 / 77$ & $78 / 67$ & N.S. \\
\hline Hypertension $(\%, n)$ & $61.2(101)$ & $61.3(117)$ & $57.8(104)$ & $60.7(88)$ & 0.893 \\
\hline Hyperlipidemia $(\%, n)$ & $10.9(18)$ & $32.3(21)$ & $8.9(16)$ & $6.9(10)$ & 0.554 \\
\hline TC (mmol/L) & $4.97 \pm 1.04$ & $4.93 \pm 0.81$ & $4.94 \pm 0.77$ & $4.93 \pm 0.90$ & 0.972 \\
\hline HDL-c (mmol/L) & $1.31 \pm 0.32$ & $1.29 \pm 0.30$ & $1.28 \pm 0.30$ & $1.30 \pm 0.31$ & 0.836 \\
\hline LDL-c (mmol/L) & $3.03 \pm 0.95$ & $3.13 \pm 2.12$ & $2.97 \pm 0.69$ & $2.97 \pm 0.77$ & 0.629 \\
\hline TG (mmol/L) & $1.68 \pm 2.08$ & $1.66 \pm 1.31$ & $1.65 \pm 1.23$ & $1.67 \pm 0.69$ & 0.997 \\
\hline Apo-A I (g/L) & $1.14 \pm 1.16$ & $1.14 \pm 0.23$ & $1.18 \pm 0.19$ & $1.16 \pm 0.16$ & 0.180 \\
\hline Apo-B (g/L) & $0.89 \pm 1.12$ & $0.89 \pm 1.13$ & $0.89 \pm 1.12$ & $0.87 \pm 1.11$ & 0.326 \\
\hline Apo-A I / Apo-B & $1.32 \pm 0.28$ & $1.32 \pm 0.33$ & $1.34 \pm 0.28$ & $1.36 \pm 0.28$ & 0.486 \\
\hline Apo-E (mg/L) & $51.6 \pm 5.23$ & $51.9 \pm 5.29$ & $51.5 \pm 4.65$ & $51.3 \pm 4.44$ & 0.647 \\
\hline Diabetes mellitus $(\%, n)$ & $24.2(40)$ & $29.3(56)$ & $22.2(40)$ & $24.1(35)$ & 0.434 \\
\hline FPG (mmol/L) & $5.53 \pm 2.14$ & $5.68 \pm 1.72$ & $5.49 \pm 1.59$ & $5.53 \pm 1.91$ & 0.754 \\
\hline PG2h (mmol/L) & $10.5 \pm 1.75$ & $10.4 \pm 1.40$ & $10.3 \pm 1.31$ & $10.2 \pm 1.36$ & 0.313 \\
\hline $\mathrm{HbA}_{1 \mathrm{c}}(\%)$ & $5.86 \pm 1.28$ & $5.86 \pm 1.17$ & $5.80 \pm 1.08$ & $5.70 \pm 1.24$ & 0.582 \\
\hline Tobacco use $(\%, n)$ & $35.8(59)$ & $31.7(79)$ & $42.2(76)$ & $21.6(59)$ & 0.619 \\
\hline cTnT (ng/ml) & $4.8 \pm 3.5$ & $4.5 \pm 3.8$ & $4.8 \pm 3.6$ & $5.0 \pm 3.8$ & 0.750 \\
\hline LVEF (\%) & $53.6 \pm 15.0$ & $54.5 \pm 11.6$ & $54.6 \pm 12.1$ & $53.7 \pm 13.4$ & 0.829 \\
\hline WMA $(\%, n)$ & $68.5(113)$ & $66.0(126)$ & $68.9(124)$ & $62.8(91)$ & 0.641 \\
\hline $\operatorname{BMI}\left(\mathrm{kg} / \mathrm{m}^{2}\right)$ & $24.5 \pm 2.1$ & $24.7 \pm 2.1$ & $24.6 \pm 1.8$ & $24.4 \pm 2.2$ & 0.601 \\
\hline Hospitalization days & $10.6 \pm 6.3$ & $10.6 \pm 5.4$ & $10.5 \pm 3.0$ & $10.5 \pm 3.8$ & 0.983 \\
\hline \multicolumn{6}{|l|}{ Drug therapy } \\
\hline Aspirin $(\%, n)$ & $85.5(141)$ & $94.2(180)$ & $93.3(168)$ & 95.9 (139) & 0.001 \\
\hline Clopidogrel $(\%, n)$ & 70.9 (117) & $93.2(178)$ & $93.3(168)$ & $93.1(135)$ & $<0.001$ \\
\hline$\beta$-blocker $(\%, \mathrm{n})$ & $61.2(101)$ & $57.1(109)$ & $68.9(124)$ & $58.6(85)$ & 0.100 \\
\hline ACEI/ARB (\%,n) & $51.5(85)$ & $50.8(97)$ & $53.3(96)$ & $62.1(90)$ & 0.169 \\
\hline Statins $(\%, n)$ & $87.3(144)$ & $89.0(170)$ & $91.1(164)$ & $89.7(130)$ & 0.715 \\
\hline Digitalis $(\%, \mathrm{n})$ & $7.3(12)$ & $5.2(10)$ & $5.6(10)$ & $3.4(5)$ & 0.528 \\
\hline Diuretics (\%, n) & $3.6(6)$ & $7.3(14)$ & $6.1(11)$ & $3.4(5)$ & 0.294 \\
\hline CCB $(\%, n)$ & $6.1(10)$ & $10.5(20)$ & $11.1(20)$ & $10.3(15)$ & 0.372 \\
\hline Nitrates $(\%, \mathrm{n})$ & $37.6(62)$ & $39.8(76)$ & $31.1(56)$ & $36.6(53)$ & 0.358 \\
\hline DAPT adherence $(\%, n)$ & $70.9(117)$ & $91.1(174)$ & $92.8(167)$ & $93.1(35)$ & $<0.001$ \\
\hline \multicolumn{6}{|c|}{ Cardiac function classification (Killip) } \\
\hline $\mathrm{I}(\%, \mathrm{n})$ & $89.1(147)$ & $89.5(171)$ & $90.6(163)$ & $89.7(130)$ & 0.975 \\
\hline II $(\%, n)$ & $6.7(11)$ & $5.8(11)$ & $3.9(7)$ & $2.8(4)$ & 0.353 \\
\hline III $(\%, n)$ & $1.8(3)$ & $1.0(2)$ & $0.6(1)$ & $4.1(6)$ & 0.078 \\
\hline IV $(\%, n)$ & $10.3(17)$ & $9.4(18)$ & $9.4(17)$ & $9.7(14)$ & 0.992 \\
\hline \multicolumn{6}{|l|}{ House Localization (km) } \\
\hline$<10 \mathrm{~km}(\%, \mathrm{n})$ & $32.7(54)$ & $31.9(61)$ & $31.7(57)$ & $32.4(47)$ & 0.997 \\
\hline $10 \mathrm{~km} \leq<20 \mathrm{~km}(\%, \mathrm{n})$ & $52.1(86)$ & $54.5(104)$ & $54.4(98)$ & $53.8(78)$ & 0.969 \\
\hline$\geq 20 \mathrm{~km}(\%, \mathrm{n})$ & $15.2(25)$ & $13.6(26)$ & $13.9(25)$ & $13.8(20)$ & 0.977 \\
\hline
\end{tabular}

Table 2. Incidence of major adverse events and cardiac mortality of the enrolled patients with different ages.

\begin{tabular}{|c|c|c|c|c|c|}
\hline Characteristics $(\% ; n)$ & NCMS group $(n=165)$ & URBMI group $(n=191)$ & UEBMI group $(n=180)$ & UNINSURED group $(\mathrm{n}=145)$ & $\mathrm{p}$ value \\
\hline Age $<65$ years & $33.9(56)^{\# \dagger}$ & $35.1(67)^{\# \dagger}$ & $95.6(172)^{*}$ & $90.3(131)^{* \mp}$ & $<0.001$ \\
\hline Major adverse events & $9.1(15)$ & $5.8(11)$ & $12.2(22)$ & $21.4(31)$ & 0.008 \\
\hline Cardiac mortality & $1.8(3)$ & $1.0(2)$ & $4.4(8)$ & $5.5(8)$ & 0.020 \\
\hline Age $\geq 65$ years & $66.1(109)^{\# \dagger}$ & $64.9(124)^{\# \dagger}$ & $4.4(8)^{\star \star}$ & $9.7(14)^{* \pi}$ & $<0.001$ \\
\hline Major adverse events & $29.7(49)$ & $18.8(36)$ & $3.3(6)$ & $6.2(9)$ & $<0.001$ \\
\hline Cardiac mortality & $9.7(16)$ & $2.6(5)$ & $0.5(1)$ & $1.4(2)$ & $<0.001$ \\
\hline
\end{tabular}

Inter-group analysis: " $p<0.05$ versus NCMS group; ${ }^{\uparrow} p<0.05$ versus URBMI group; ${ }^{\#} \mathrm{p}<0.05$ versus UEBMI group; ${ }^{\dagger} \mathrm{p}<0.05$ versus UNINSURED group.

Abbreviation: NCMS new rural cooperative medical scheme, URBMI urban resident basic medical insurance scheme, UEBMI urban employee basic medical insurance scheme. 
$\mathbf{A}$

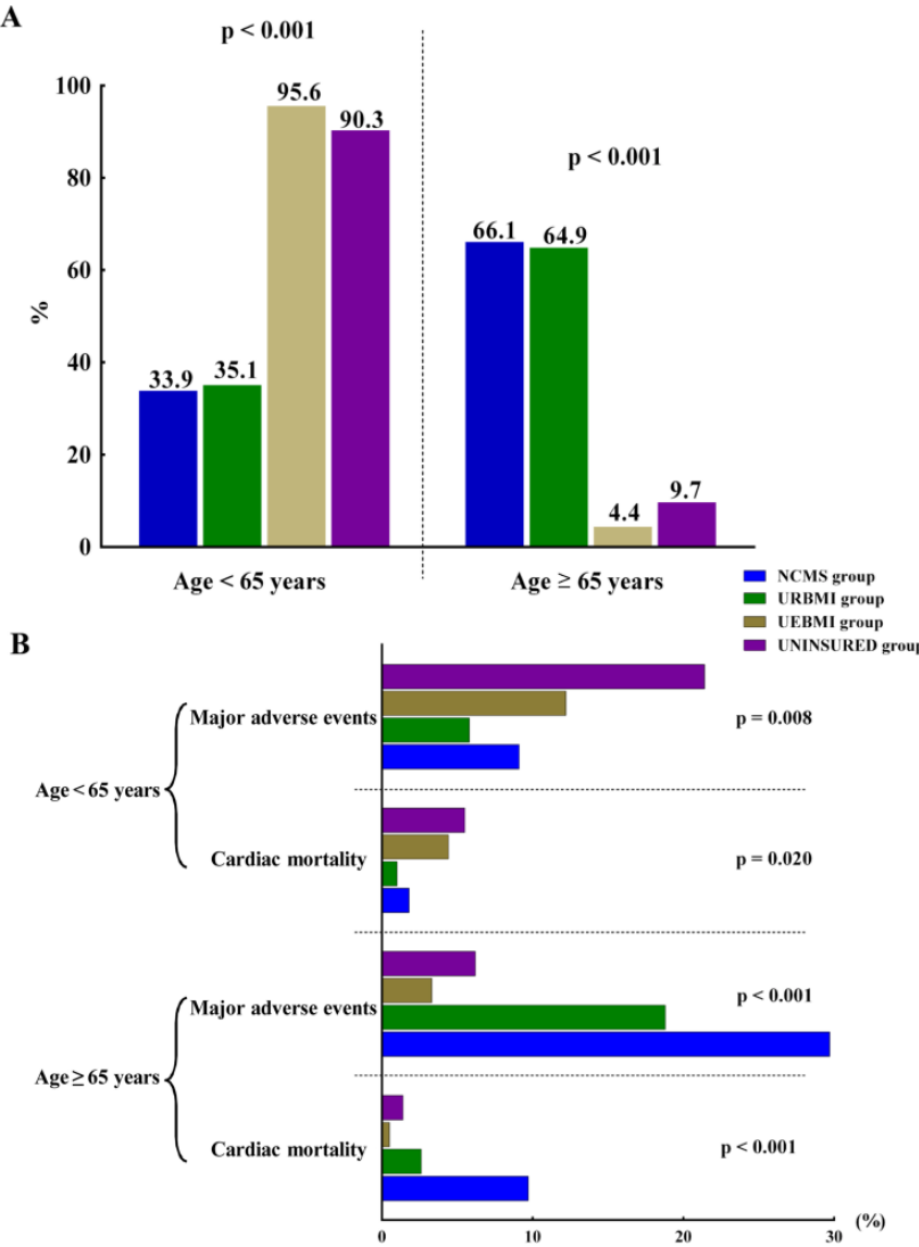

Figure 2. Incidence of major adverse events and cardiac mortality of the enrolled patients with different ages. (A) The proportion of patients with an age $<65$ years or $\geq 65$ years in four groups. (B) Incidence of major adverse events and cardiac mortality of the enrolled patients with different ages. The proportion of patients $\geq 65$ years with a major adverse event was $29.7 \%$ and $18.8 \%$ in NCMS group and URBMI group, respectively. However, this proportion was significantly lower in UEBMI and UNINSURED groups (3.3\% and $6.2 \%$, respectively). The majority of patients with a major adverse event in UEBMI and UNINSURED groups were $<65$ years. Abbreviations: NCMS, new rural cooperative medical scheme; URBMI, urban resident basic medical insurance scheme; UEBMI, urban employee basic medical insurance scheme.

\section{Angiographic results}

All enrolled patients were confirmed by coronary angiography (CAG). Data on coronary lesions and stents were compared. Multiple views of each coronary artery were obtained and all images of the coronary tree were recorded as appropriate and reproduced at the time of follow-up angiography. The initial and follow-up coronary angiograms were obtained in the same projection, and the images were quantitatively assessed by a single cardiologist.

All the CAG results in four groups were presented in Table 3. Adachi/Bianchi classification was used to categorize the particular vasculature types: classic coronary artery, neither artery is dominating; dominant right coronary artery; dominant left coronary artery [19]. The severity of stenosis was graded by using the Coronary Angiogram Analyzing System II (CAAS II; Pie Medical, Maastricht, The Netherlands) [20]. Preprocedural and postprocedural coronary flow over the culprit lesion was graded according to the Thrombolysis in Myocardial Infarction Trial (TIMI) criteria, and collateral circulation was classified according to the criteria proposed by Rentrop et al. [21-23]. Multivessel CAD was defined as the presence of lesions in $\geq 3$ coronary vessels. Single-vessel disease referred to occlusion in the main and secondary branch of a vessel [24].

Among the enrolled patients, 35 patients showed dominant left coronary artery, 562 showed dominant right coronary artery, and 84 showed classic coronary artery. 157 patients were with single vessel disease, 246 with double vessels disease and 278 with three vessels disease. The proportion of patients with double vessels disease was the highest in URBMI group ( $p=0.024$, Figure 3 ). The incidence of three vessels disease was the highest in the UNINSURED group ( $p=0.001$, Figure 3 ). Similarly, severe and mild degrees of coronary stenosis showed significant difference among the enrolled patients $(p=0.047$ and $p=0.036$, respectively).

The door-to-balloon time of the enrolled patients was 101.8 \pm 9.4 minutes, which was still more than 90 minutes the Guideline recommended. The door-to-balloon time showed no statistical significance in four groups ( $p=0.684$, Table 3 ). However, the symptom-to-balloon time in NCMS group was $240.5 \pm 8.89$ minutes, which was significantly higher than the other groups $(\mathrm{p}<0.001$, Table 2$)$. 
Table 3. Comparison of coronary angiography results of enrolled patients.

\begin{tabular}{|c|c|c|c|c|c|}
\hline & NCMS group $(n=165)$ & URBMI group $(n=191)$ & UEBMI group $(n=180)$ & $\begin{array}{l}\text { UNINSURED group } \\
(\mathrm{n}=145)\end{array}$ & p value \\
\hline \multicolumn{6}{|l|}{ Coronary lesions } \\
\hline Single-vessel disease (n) & 36 & 44 & 44 & 33 & 0.142 \\
\hline 2-vessel disease (n) & 50 & 78 & 72 & 46 & 0.024 \\
\hline 3-vessel disease (n) & 79 & 69 & 64 & 66 & 0.001 \\
\hline \multicolumn{6}{|l|}{ Degree of coronary stenosis } \\
\hline $90 \%<\mathrm{D} \leq 100 \%$ (n) & 59 & 68 & 64 & 47 & 0.165 \\
\hline $70 \%<\mathrm{D} \leq 90 \%(\mathrm{n})$ & 152 & 165 & 153 & 151 & 0.036 \\
\hline $50 \% \leq \mathrm{D} \leq 70 \%(\mathrm{n})$ & 162 & 164 & 163 & 125 & 0.047 \\
\hline \multicolumn{6}{|l|}{ Type of coronary vasculature } \\
\hline Dominant RCA (n) & 136 & 163 & 148 & 115 & 0.552 \\
\hline Classic (n) & 23 & 16 & 20 & 25 & 0.084 \\
\hline Dominant LCA (n) & 6 & 12 & 12 & 5 & 0.393 \\
\hline \multicolumn{6}{|l|}{ Residual stenosis } \\
\hline Non-reperfusion (n) & 10 & 23 & 12 & 10 & 0.127 \\
\hline$\leq 20 \%(\mathrm{n})$ & 120 & 133 & 144 & 105 & 0.137 \\
\hline$>20 \%(\mathrm{n})$ & 35 & 35 & 24 & 30 & 0.215 \\
\hline \multicolumn{6}{|l|}{ Preprocedural TIMI flow } \\
\hline $0(\mathrm{n})$ & 176 & 183 & 180 & 158 & 0.407 \\
\hline $1(\mathrm{n})$ & 92 & 100 & 91 & 76 & 0.143 \\
\hline $2(\mathrm{n})$ & 86 & 91 & 89 & 71 & 0.363 \\
\hline $3(\mathrm{n})$ & 19 & 23 & 20 & 18 & 0.538 \\
\hline \multicolumn{6}{|l|}{ Postprocedural TIMI flow } \\
\hline $2(\mathrm{n})$ & 15 & 21 & 16 & 11 & 0.163 \\
\hline $3(n)$ & 294 & 301 & 286 & 262 & 0.108 \\
\hline \multicolumn{6}{|l|}{ Revascularization } \\
\hline PTCA (n) & 32 & 35 & 33 & 40 & 0.130 \\
\hline PTCA+Stent (n) & 123 & 133 & 135 & 95 & 0.196 \\
\hline CAG only (n) & 10 & 23 & 12 & 10 & 0.127 \\
\hline Number of stents (n) & 155 & 167 & 168 & 110 & N.S. \\
\hline Stent diameter (mm) & $3.15 \pm 0.51$ & $3.06 \pm 0.42$ & $3.11 \pm 0.54$ & $3.09 \pm 0.43$ & 0.402 \\
\hline Stent length (mm) & $28.42 \pm 13.78$ & $29.34 \pm 15.55$ & $27.32 \pm 10.89$ & $26.74 \pm 10.86$ & 0.418 \\
\hline Lesion length (mm) & $29.11 \pm 13.74$ & $30.12 \pm 15.38$ & $28.07 \pm 11.06$ & $27.39 \pm 10.70$ & 0.388 \\
\hline Door-to-balloon time (min) & $102.5 \pm 9.24$ & $101.4 \pm 8.95$ & $102.0 \pm 10.28$ & $101.4 \pm 8.91$ & 0.684 \\
\hline Symptom-to-balloon time (min) & $240.5 \pm 8.89$ & $235.1 \pm 8.81$ & $237.0 \pm 9.16$ & $236.8 \pm 9.09$ & $<0.001$ \\
\hline
\end{tabular}

Abbreviation: NCMS new rural cooperative medical scheme, URBMI urban resident basic medical insurance scheme, UEBMI urban employee basic medical insurance scheme, RCA right coronary artery, LCA left coronary artery, D diameter, N.S. not suited, CAG coronary angiography, PTCA percutaneous transluminal coronary angioplasty, TIMI Thrombolysis in Myocardial Infarction Trial criteria.

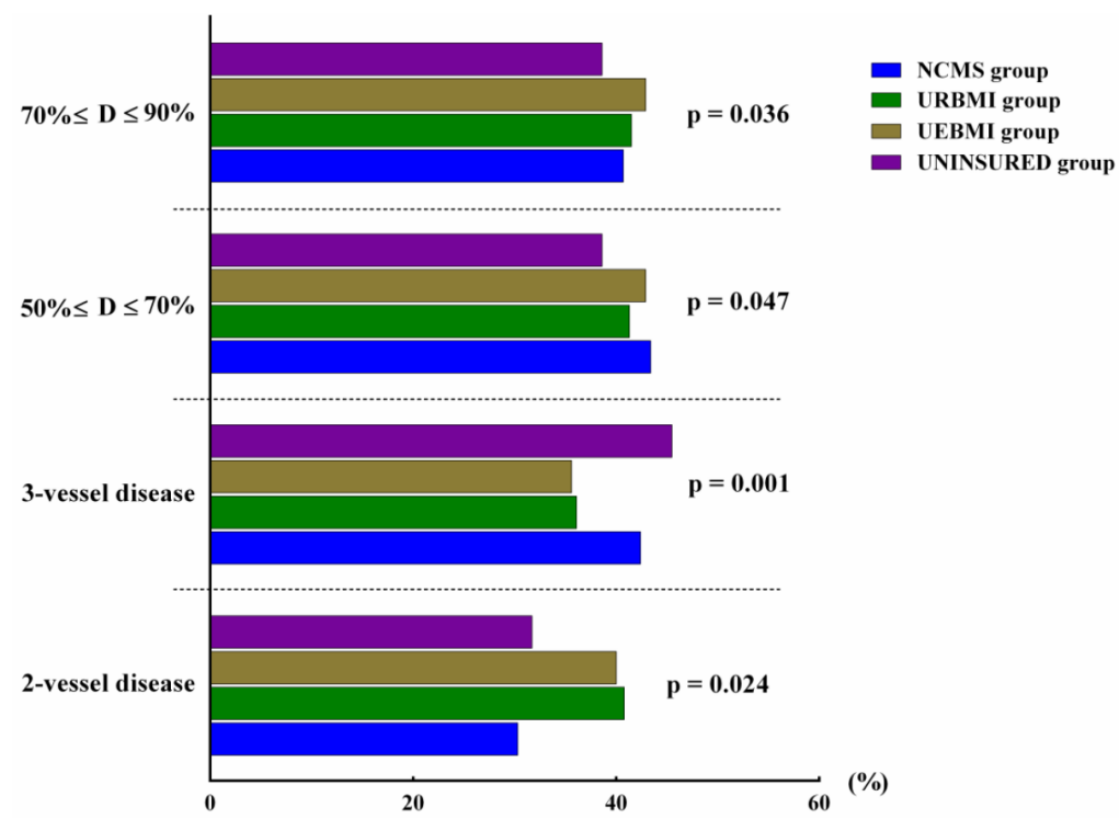

Figure 3. The comparison of coronary lesions and coronary stenosis of the enrolled patients reached statistical significance. Abbreviations: $D$, diameter; BSMI, basic social medical insurance; NCMS, new rural cooperative medical scheme; URBMI, urban resident basic medical insurance scheme; UEBMI, urban employee basic medical insurance scheme. 


\section{Analysis of economic factors}

In the study, BSMI-related economic factors were collected: household income per year, gross medical cost, out-of-pocket medical cost and commercial health insurance. Household income and gross medical cost were directly collected from the patients, the out-of-pocket cost were calculated according to the policies aforementioned. The gross medical cost was classified into three categories $(<25 \mathrm{~K}, 25 \mathrm{~K} \leq<50 \mathrm{~K}$,

A
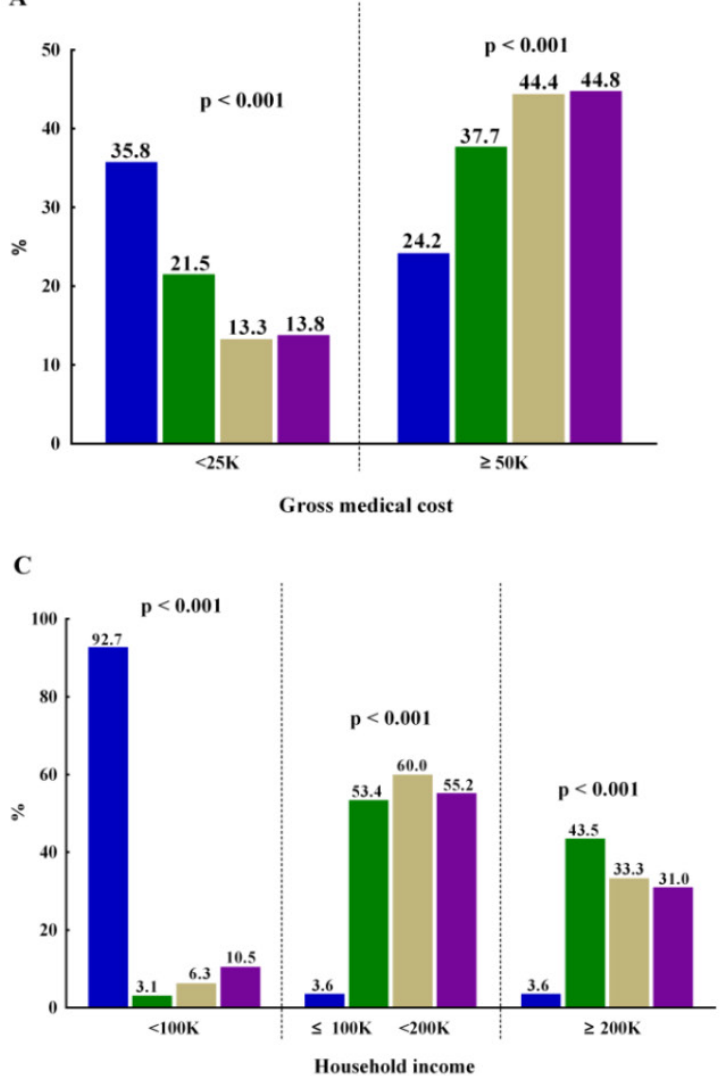

$\geq 50 \mathrm{~K}$, all in RMB). Likewise, out-of-pocket cost and household income were also classified into three categories $([<10 \mathrm{~K}, 10 \mathrm{~K} \leq<20 \mathrm{~K}, \geq 20 \mathrm{~K}]$ and $[<100 \mathrm{~K}, 100 \mathrm{~K} \leq$ $<200 \mathrm{~K}, \geq 200 \mathrm{~K}]$, respectively).

Analysis of economic factors was presented in Table 4. The gross medical cost of the NCMS group was relatively lower compared with the other patients $(\mathrm{p}<0.001$, Figure 4).

B

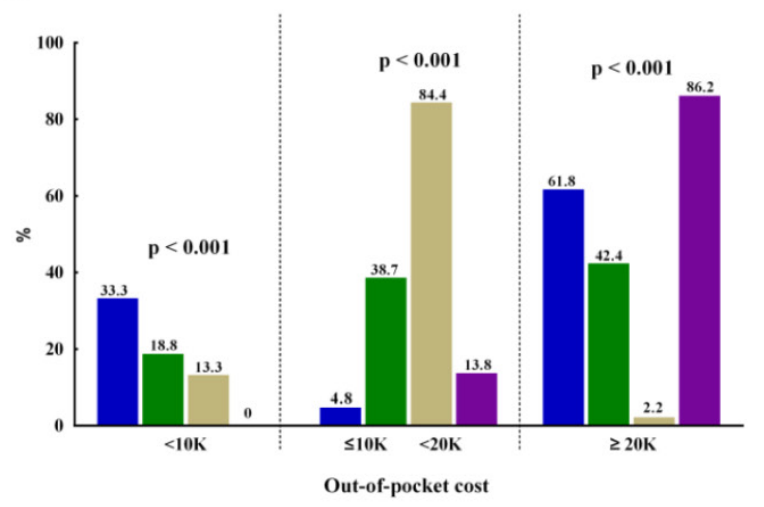

D
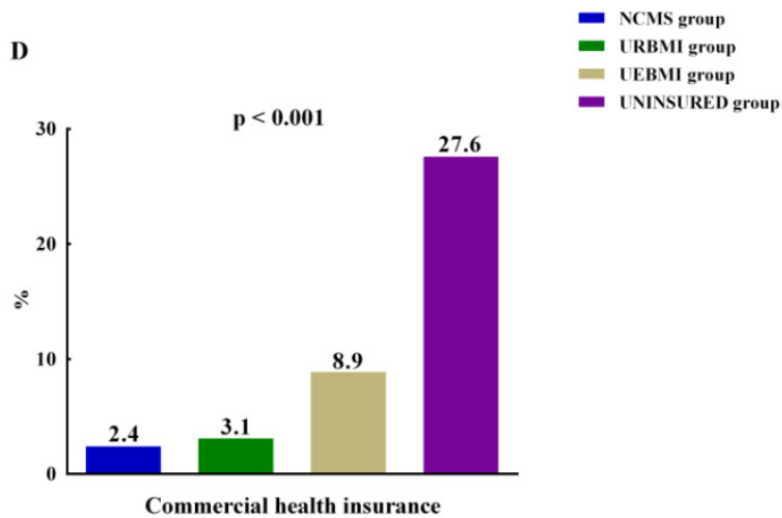

Figure 4. The comparison of economic factors reached statistical significance in four groups. (A) Comparison of gross medical cost in four groups. (B) Comparison of out-of-pocket cost in four groups. (C) Comparison of household income in four groups. (D) Distribution of commercial health insurance in four groups. Abbreviations: NCMS, new rural cooperative medical scheme; URBMI, urban resident basic medical insurance scheme; UEBMI, urban employee basic medical insurance scheme.

Table 4. Comparison of economic factors in four groups.

\begin{tabular}{|c|c|c|c|c|c|}
\hline & $\begin{array}{l}\text { NCMS group } \\
(n=165)\end{array}$ & $\begin{array}{l}\text { URBMI group } \\
(n=191)\end{array}$ & $\begin{array}{l}\text { UEBMI group } \\
(\mathrm{n}=180)\end{array}$ & $\begin{array}{l}\text { UNINSURED group } \\
(\mathrm{n}=145)\end{array}$ & $\mathrm{p}$ value \\
\hline Gross medical cost $(\mathrm{K})$ & $36.2 \pm 19.5$ 什 & $43.4 \pm 18.6^{*} \dagger$ & $45.5 \pm 19.3^{*+}$ & $51.4 \pm 21.6^{\star \star \varpi \# ~}$ & $<0.001$ \\
\hline Gross medical cost $(<25 K)(\%, n)$ & $35.8(59)$ & $21.5(41)$ & $13.3(24)$ & $13.8(20)$ & \\
\hline Gross medical cost $(25 \mathrm{~K} \leq<50 \mathrm{~K})(\%, \mathrm{n})$ & $40.0(66)$ & $40.8(78)$ & $42.2(76)$ & $41.4(60)$ & \\
\hline Gross medical cost $(\geq 50 K)(\%, n)$ & $24.2(40)$ & $37.7(72)$ & $44.4(80)$ & $44.8(65)$ & \\
\hline Out-of-pocket cost (K) & $18.1 \pm 9.8^{\# \dagger}$ & $18.3 \pm 8.1^{\#+}$ & $13.4 \pm 2.8^{* \oplus \dagger}$ & $51.4 \pm 21.6^{\star \star \varpi \# ~}$ & $<0.001$ \\
\hline Out-of-pocket cost $(<10 K)(\%, n)$ & $33.3(55)$ & $18.8(36)$ & $13.3(24)$ & 0 & \\
\hline Out-of-pocket cost $(10 \mathrm{~K} \leq<20 \mathrm{~K})(\%, \mathrm{n})$ & $4.8(8)$ & $38.7(74)$ & $84.4(152)$ & $13.8(20)$ & \\
\hline Out-of-pocket cost $(\geq 20 \mathrm{~K})(\%, \mathrm{n})$ & $61.8(102)$ & $42.4(81)$ & $2.2(4)$ & $86.2(125)$ & \\
\hline \multicolumn{6}{|l|}{ Household income } \\
\hline Household income $(<100 \mathrm{~K})(\%, \mathrm{n})$ & $92.7(153)^{\curvearrowleft}$ 『† & $3.1(6)^{* \# \dagger}$ & $6.3(12)^{\star \star \top} \mid$ & $10.5(20)^{\star \star \# \#}$ & $<0.001$ \\
\hline Household income $(100 \mathrm{~K} \leq<200 \mathrm{~K})(\%, \mathrm{n})$ & $3.6(6)^{\text {9\#† }}$ & $53.4(102)^{*}$ & $60.0(108)^{*}$ & $55.2(80)^{*}$ & $<0.001$ \\
\hline Household income $(\geq 200 K)(\%, n)$ & $3.6(6)^{\natural} \#+$ & $43.5(83)^{\star \# \dagger}$ & $33.3(60)^{*}$ & $31.0(45)^{\star \star ा ~}$ & $<0.001$ \\
\hline Commercial health insurance $(\%, n)$ & $2.4(4)^{\# \dagger}$ & $3.1(6)^{\# \dagger}$ & $8.9(16)^{\star \star \top \dagger}$ & $27.6(40)^{\star \star \circledast \# ~}$ & $<0.001$ \\
\hline
\end{tabular}

Inter-group analysis: ${ }^{\mathrm{p}} \mathrm{p}<0.05$ versus NCMS group; ${ }^{\uparrow} \mathrm{p}<0.05$ versus URBMI group; ${ }^{\#} \mathrm{p}<0.05$ versus UEBMI group; ${ }^{\dagger} \mathrm{p}<0.05$ versus UNINSURED group.

Abbreviation: NCMS new rural cooperative medical scheme, URBMI urban resident basic medical insurance scheme, UEBMI urban employee basic medical insurance scheme. 
The out-of-pocket cost showed significantly difference in the study groups. The UNINSURED patients should pay all gross medical cost and thus were sorted into $\geq 20 \mathrm{~K}$ category mostly $(125 ; 86.2 \%)$. As to BSMI participants, majority of UEBMI patients' out-of-pocket cost (n; \%) was in the $10 \mathrm{~K} \leq<20 \mathrm{~K}$ category $(152 ; 84.4 \%)$. Although supported by BSMI, most patients in NCMS group still paid $\geq 20 \mathrm{~K}$ after insurance reimbursement (102; 61.8\%) (Figure 4). The household income of NCMS patients (n; \%) were mainly in $<100 \mathrm{~K}$ category (153; 92.7\%), which differs significantly from the other groups $(\mathrm{p}<0.001)$ (Figure 4). Commercial health insurance covered significantly more patients in UNINSURED group (40; 27.6\%, $\mathrm{p}<0.001$ ) compared with other groups (Figure 4).

\section{BSMI as an important factor in clinical outcomes}

The mean time of follow-up was $31.3 \pm 9.3$ months. The cumulative incidence of major adverse events was outlined in Table 5. Cardiac mortality (n; $\%)$ was the highest in the NCMS group $(19 ; 11.5 \%)$ and the lowest in URBMI group (7; 3.7\%) (Figure 5). Inter-group analysis found that cardiac mortality in NCMS group showed significant difference when compared with other groups (all $\mathrm{p}<0.05$ ). The proportion of patients with a major adverse event (n; \%) was also the highest in the NCMS group $(64 ; 38.8 \%$ ) but the lowest in UEBMI group (28; 15.6\%). Significantly higher incidence of a major adverse event was also in NCMS group $(p<0.001)$ (Figure 5). A Kaplan-Meier survival analysis for cardiac mortality showed lower event-free survival for the NCMS group (mean 33.9 months, 95\% CI 33.0-34.9, p=0.007; Figure 6). Similar$1 \mathrm{y}$, the Kaplan-Meier survival analysis for major adverse events revealed significantly lower event-free survival in the NCMS group as compared with the other three groups (mean 28.9 months, 95\% CI 27.2-30.5 p<0.001; Figure 6).

A
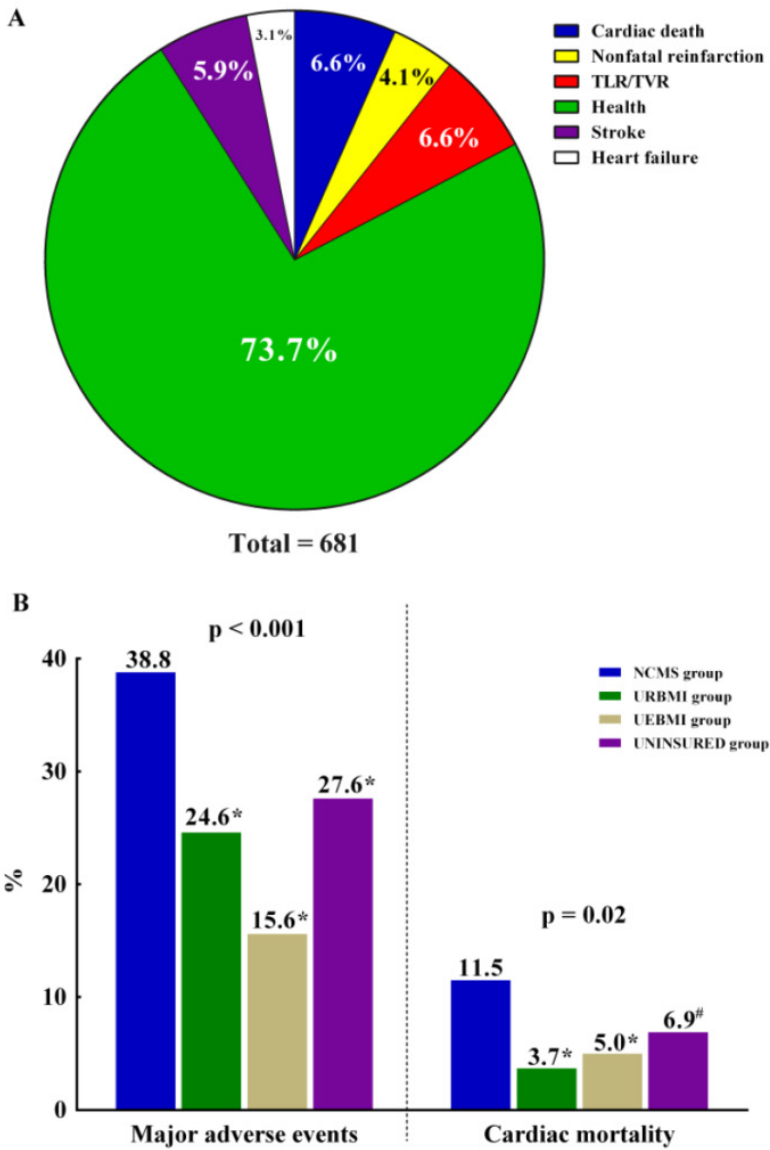

Figure 5. The clinical outcomes of the enrolled patients. (A) The proportion of different major adverse events of the enrolled patients. (B) Cardiac mortality and incidence of major adverse events in four groups. The incidence of major adverse events was significantly higher in NCMS patients $(64 ; 38.8 \%)$ compared with the other groups: URBMI (47; 24.6\%); UEBMI (28; 15.6\%); UNISURED (40; 27.6\%). Similarly, cardiac mortality was also higher in NCMS group (19; $11.5 \%)$ compared with the other groups: URBMI (7; 3.7\%); UEBMI (9; 5.0\%); UNISURED (I0;6.9\%). * $p<0.001$ versus NCMS group, $\# p<0.05$ versus NCMS group. Abbreviations: TLR, target lesion revascularization; TVR, target vessel revascularization; NCMS, new rural cooperative medical scheme; URBMI, urban resident basic medical insurance scheme; UEBMI, urban employee basic medical insurance scheme.
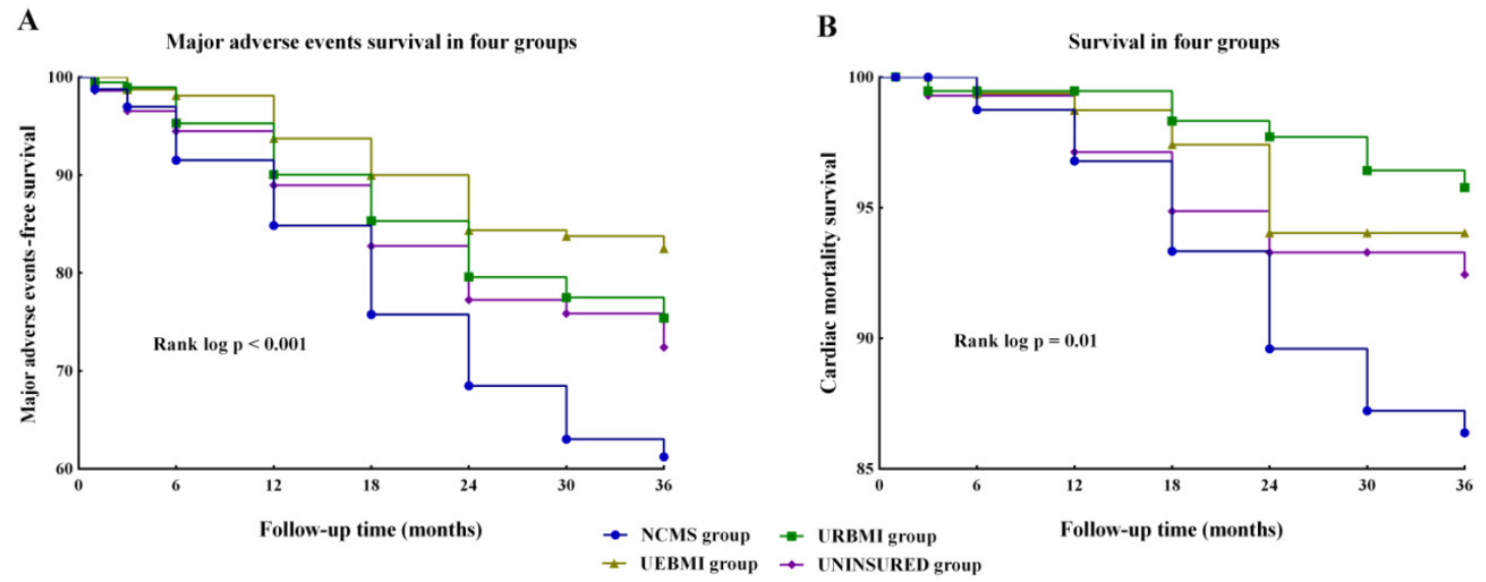

Figure 6. Kaplan-Meier analysis showing cardiac mortality and major adverse events in four groups. (A) Kaplan-Meier analysis of major adverse events in four groups. (B) Kaplan-Meier analysis of survival in four groups. Abbreviations: NCMS, new rural cooperative medical scheme; URBMI, urban resident basic medical insurance scheme; UEBMI, urban employee basic medical insurance scheme. 
Table 5. The incidence of major adverse events of the enrolled patients.

\begin{tabular}{|c|c|c|c|c|c|}
\hline & $\begin{array}{l}\text { NCMS } \\
\text { group } \\
(n=165)\end{array}$ & $\begin{array}{l}\text { URBMI } \\
\text { group } \\
(n=191)\end{array}$ & $\begin{array}{l}\text { UEBMI } \\
\text { group } \\
(n=180)\end{array}$ & $\begin{array}{l}\text { UNINSURED } \\
\text { group } \\
(n=145)\end{array}$ & $\begin{array}{l}\mathrm{p} \\
\text { value }\end{array}$ \\
\hline Cardiac death $(\%, \mathrm{n})$ & $11.5(19)$ & $3.7(7)$ & $5.0(9)$ & $6.9(10)$ & 0.020 \\
\hline Nonfatal reinfarction $(\%, \mathrm{n})$ & $6.7(11)$ & $4.7(9)$ & $2.2(4)$ & $2.8(4)$ & 0.157 \\
\hline $\operatorname{TLR} / \operatorname{TVR}(\%, \mathrm{n})$ & $8.5(14)$ & $8.4(16)$ & $1.7(3)$ & $8.3(12)$ & 0.021 \\
\hline Stroke $(\%, \mathrm{n})$ & $7.3(12)$ & $5.8(11)$ & $4.4(8)$ & $6.2(9)$ & 0.733 \\
\hline Heart failure $(\%, n)$ & $4.8(8)$ & $2.1(4)$ & $2.2(4)$ & $3.4(5)$ & 0.414 \\
\hline
\end{tabular}

The multivariate Cox regression analysis showed a significant association between BSMI and major adverse events after adjusting for other relevant factors: age, gender, BMI, hypertension, hyperlipidemia, cTnT, WMA, diabetes mellitus, tobacco use, LVEF, DAPT adherence and economic factors (Table 6). Age, BSMI, cTnT, DAPT adherence and out-of-pocket cost were all considered important factors for patients who had significantly lower event-free survival for major adverse events (Figure 7). Analysis for cardiac mortality showed that BSMI may not be an independent factor to predict cardiac death after adjusting for other risk factors, including age, gender, BMI, hypertension, cTNT, WMA, hyperlipidemia, diabetes mellitus, tobacco use, LVEF, DAPT adherence and economic factors. However, the DAPT adherence was an independent predictor for cardiac death.

Table 6. Results of multivariate Cox regression analysis with all-cause cardiac mortality and major adverse events.

\begin{tabular}{|c|c|c|c|c|c|c|c|c|}
\hline \multirow{3}{*}{ Characteristics } & \multicolumn{4}{|c|}{ Major adverse events } & \multicolumn{4}{|c|}{ Cardiac death } \\
\hline & \multirow[t]{2}{*}{ Hazard } & \multicolumn{2}{|c|}{$95.0 \% \mathrm{CI}$} & \multirow[t]{2}{*}{$\mathrm{p}$ value } & \multirow[t]{2}{*}{ Hazard } & \multicolumn{2}{|c|}{$95.0 \% \mathrm{CI}$} & \multirow[t]{2}{*}{$\mathrm{p}$ value } \\
\hline & & Lower & Upper & & & Lower & Upper & \\
\hline Age & 1.981 & 1.966 & 1.996 & 0.016 & 1.031 & 0.985 & 1.079 & 0.195 \\
\hline Gender & 0.874 & 0.648 & 1.178 & 0.376 & 0.570 & 0.305 & 1.067 & 0.079 \\
\hline BMI & 1.058 & 0.977 & 1.145 & 0.165 & 1.507 & 1.189 & 1.909 & 0.001 \\
\hline BSMI & 0.713 & 0.566 & 0.897 & 0.004 & 1.839 & 0.897 & 3.770 & 0.096 \\
\hline cTNT & 0.955 & 0.913 & 0.998 & 0.040 & 1.010 & 0.900 & 1.132 & 0.871 \\
\hline LVEF & 1.009 & 0.996 & 1.023 & 0.158 & 0.995 & 0.968 & 1.022 & 0.707 \\
\hline WMA & 0.668 & 0.440 & 1.015 & 0.059 & 0.550 & 0.159 & 1.901 & 0.345 \\
\hline Hypertension & 0.815 & 0.582 & 1.141 & 0.233 & 0.546 & 0.213 & 1.400 & 0.208 \\
\hline Diabetes & 0.826 & 0.579 & 1.177 & 0.290 & 1.188 & 0.551 & 2.559 & 0.660 \\
\hline Hyperlipidemia & 0.665 & 0.415 & 1.066 & 0.090 & 0.666 & 0.250 & 1.774 & 0.666 \\
\hline Tobacco use & 0.754 & 0.553 & 1.029 & 0.075 & 0.563 & 0.275 & 1.153 & 0.116 \\
\hline DAPT adherence & 0.267 & 0.181 & 0.394 & $<0.001$ & 0.041 & 0.016 & 0.103 & $<0.001$ \\
\hline Household income & 0.982 & 0.750 & 1.285 & 0.892 & 0.616 & 0.288 & 1.319 & 0.212 \\
\hline Commercial health insurance & 0.848 & 0.450 & 1.598 & 0.611 & 1.511 & 0.899 & 2.727 & 0.352 \\
\hline Gross medical cost & 0.983 & 0.817 & 1.141 & 0.900 & 0.791 & 0.655 & 0.951 & 0.029 \\
\hline Out-of-pocket cost & 0.877 & 0.761 & 0.992 & 0.019 & 0.972 & 0.794 & 1.179 & 0.312 \\
\hline
\end{tabular}

Abbreviations: NCMS new rural cooperative medical scheme, URBMI urban resident basic medical insurance scheme, UEBMI urban employee basic medical insurance scheme, cTNT cardiac troponin T, LVEF left ventricular ejection fraction, BMI body mass index, BSMI basic social medical insurance, WMA wall motion abnormality, DAPT dual anti-platelet therapy.

A

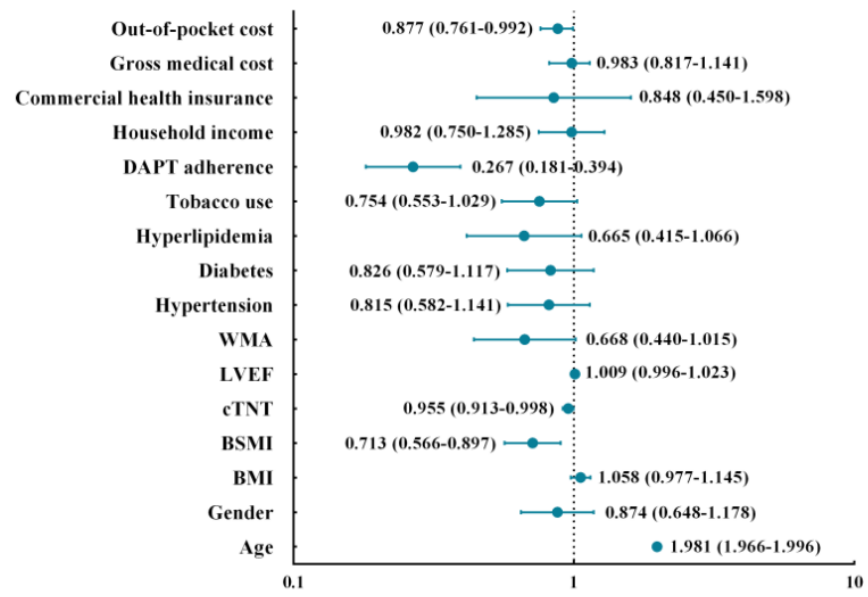

Primary outcome of major adverse events

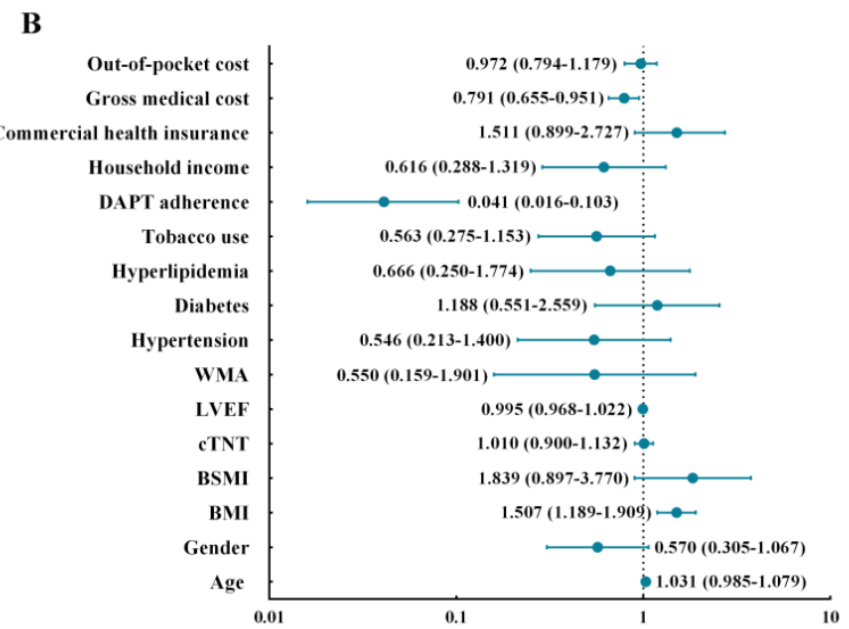

Primary outcome of cardiac mortality

Figure 7. Primary outcome of major adverse events and cardiac mortality. (A) Multivariate Cox regression analysis identified that age, BSMI, cTnT, DAPT adherence and out-of-pocket cost are independent correlates of 3-year major adverse events. (B) Multivariate Cox regression analysis revealed that BMI, DAPT adherence and gross medical cost were all considered were all considered important factors for cardiac mortality. The $95 \%$ confidence intervals of the hazard ratios were reported for all of the significant risk factors. Abbreviations: MACE, major adverse cardiac events; BMI, body mass index; BSMI, basic social medical insurance; cTnT, cardiac troponin T; LVEF, left ventricular ejection fraction; WMA, wall motion abnormality; DAPT, dual anti-platelet therapy. 


\section{Discussion}

In this retrospective study to assess the influence of BSMI on the clinical outcomes in STEMI patients, approximately $11.5 \%$ of patients with NCMS died during a median follow-up of 36 months. For those who were enrolled in BSMI, the out-of-pocket medical cost of inpatient treatments may be relatively unacceptable for the NCMS patients with a lower household income. Even if adjusting for other cardiac risk and economic factors, age, BSMI, cTnT, DAPT adherence and out-of-pocket cost were statistically associated with the recurrence of myocardial infarction, revascularization, stroke or heart failure in patients with STEMI. Thus, BSMI plays an important role in clinical outcomes of patients with STEMI. Therefore, our study confirmed that BSMI is an independent predictor of major adverse events $(\mathrm{p}<0.001)$ in STEMI patients during a mean follow-up of 36 months, especially in the rural areas. Although BSMI is not an independent factor to predict cardiac death after adjusting for other factors, it could be served as a supplementary factor in the evaluation of the cardiac mortality in STEMI patients.

STEMI is a serious cardiovascular disorder with high morbidity and mortality, all factors influencing the therapy and outcomes should be taken into consideration and comprehensive clinical treatment methods must be established. In China, demographics, previous $\mathrm{CAD}$, symptoms and cognitive factors of patients are potentially associated with clinical outcome of STEMI patients [2,3]. However, few studies have attached economic factors to STEMI. The most important contribution of this study was the demonstration of a direct relationship between BSMI and clinical outcomes in STEMI patients in nowadays Shanghai, which links the disease with another social economic factor. In our study, DAPT adherence is an independent predictor of major adverse events and cardiac death. DAPT is necessary for the STEMI patients who received reperfusion therapy, which is also an important source of medical cost in the meantime. Since STEMI is a serious disease, the medical cost of DAPT in outpatient clinics could be partly reimbursed by BSMI. For the UEBMI patients, insurance reimbursement percentage was higher, and the DAPT adherence in UEBMI group was better $(167 ; 92.8 \%)$, the proportion of a major adverse event was also lower $(28 ; 15.6 \%)$. However, according to the current policies of BSMI in Shanghai, the insurance reimbursement percentage is relatively lower for NCMS patients. The DAPT medication was then turned into huge economic burden to some NCMS patients with lower reimbursement and they were less adherent to scheduled DAPT medication, the cardiac mortality and incidence of cardiac events were significantly higher. Therefore, we may infer that the different insurance reimbursement of BSMI could influence the DAPT adherence which finally results in different outcomes in STEMI patients. Moreover, the door-to-balloon time was similar in four groups; however, the symptom-to-balloon time was significant higher in the NCMS group. And this may not be caused by the distance from house localization to hospital which presented no significant difference in the enrolled patients (all p>0.05, Table 1). This delay in the choice of therapy may be partly attributed to the different medical cost which should be taken into consideration at first for NCMS patients.

The second important contribution of this study was providing current situation of China's health care reform to some extent, which has been ongoing for several years [25]. In our study, UEBMI covers all urban employees who were still in work and not retired. All the patients in UNINSURED groups were rural migrant workers without BSMI, which were called "the third population cohort". URBMI mainly covers urban retired residents and NCMS mainly covers the rural elderly patients in our study, thus UEBMI and UNINSURED patients were relatively younger than the patients in the other two groups. Although the patients in UEBMI and UNINSURED groups were mostly <65 years, which were much younger than that of NCMS group and URBMI group, they are also likely to develop cardiovascular diseases. Age $\geq 65$ years may not be an appropriate risk factor for these populations in our study. The high pressure to survive, long hours work time, the very basic living condition and inattention to health insurance may influence their health status [26]. Despite the door-to-balloon time has been improved significantly in the past few years [27], additional strategies are needed since the door-to-balloon time was still far from $<90 \mathrm{~min}$ the Guideline recommended [1]. What's more, the health inequalities were increasing in recent years in China and urban-rural health inequality should be properly tackled in the reform of health care [28]. The disparity in insurance reimbursement of BSMI was one of these inequalities which cannot be ignored [29]. With the further reform on health equity, the influence of BSMI on STEMI patients may be changed.

Another interesting thing we found in our study is that the UNINSURED patients were not with the high cardiac mortality and incidence of cardiac events as we formerly expected since the patients were not reimbursed by BSMI. Although the medical cost for UNINSUERD patients was huge, the economic burden may be partly relieved by commercial health insurance. The importance of commercial health insur- 
ance has been pointed out in several studies [30, 31]. While the BSMI has broad coverage in China population, the third population cohort, some 420 million rural migrant workers, was completely left out of the BSMI prior to 2007 [32]. According to the regulations of the Chinese and Shanghai governments, migrant workers of different occupations in Shanghai should all be entitled to UEBMI or NCMS [33]. However, rural migrant workers are not really covered by the BSMI system mainly because of their lack of permanent resident status or formal employment in Shanghai [34]. Moreover, unwillingness to make contributions to BSMI, different education levels, the strict reimbursement limitations on the hospital locations and the lack of the supervisory mechanisms for the policy implementation could all result in the low utilization of BSMI among this population [26, 33, 35]. Since the propaganda dynamics of commercial health insurance are attractive and the reimbursement is substantial, most rural migrant workers are more likely to choose commercial health insurance $[9,28]$. Thus the emerging health inequality among populations in urban areas was partly relieved.

All the findings in our study were not only a great help for us to find a refined risk stratification for STEMI patients, but also a suggestion for the China's reform of health care in the future. Rural and urban areas are still treated quite differently-politically, economically, and socially. Similar social distinctions also apply across different urban regions of China. In the long run, it would be desirable to integrate urban and rural residents into one scheme by merging the different schemes [8]. With the elimination of health inequalities by the reform, the influence of BSMI may be alleviated gradually. The commercial health insurance also functions markedly and the real significance of commercial health insurance in the outcome of STEMI patients' needs to be explored further.

Several limitations of this study should be recognized. The study was a single-center clinical research with a small sample size, and the follow-up duration was also relative short. We did not examine the educational levels of the enrolled patients, which may also influence the evaluation of relationship between BSMI and cardiac mortality in STEMI patients, since the educational levels of the patients may be different. Although the function of commercial health insurance has been reported in some previous studies $[30,31]$, we did not investigate the reimbursement funded by different non-government providers. The influence of commercial health insurance on the outcome of STEMI patients was only a preliminary estimate from our data. Since there are regional differences in BSMI, our study was only the reflection of BSMI in Shanghai city. Despite the limitations of our approach, the masked tests of this study provided effective proof that the BSMI, a very potent social economic factor, is independent of other known risk factors in the outcomes of STEMI.

\section{Conclusion}

Although the BSMI was not an independent risk factor in prediction of cardiac death in our study, the current data still showed that BSMI is a useful prognosis factor of major adverse events in the STEMI patients under current policies issued by Shanghai government in China, especially for the patients in rural areas.

\section{Abbreviations}

STEMI: ST-elevation myocardial infarction; CAD: coronary artery disease; ECG: electrocardiogram; PTCA: percutaneous transluminal coronary angioplasty; PCI: percutaneous coronary intervention; CABG: coronary artery bypass grafting; BSMI: basic social medical insurance; NCMS: new rural cooperative medical scheme; UEBMI: urban employee basic medical insurance scheme; URBMI: urban resident basic medical insurance scheme; TLR: revascularization; TVR: target vessel revascularization; cTNT:cardiac troponin T; LVEF: left ventricular ejection fraction; BMI: body mass index; ACEI: angiotensin converting enzyme inhibitors; ARB: angiotensin II receptor antagonists; CCB: calcium channel blocker; WMA: wall motion abnormality; DAPT: dual anti-platelet therapy; TC: total cholesterol; HDL: high density lipoprotein; LDL: low density lipoprotein; TG: triglyceride; Apo: apolipoprotein; FPG: fasting plasma glucose; PG2h: 2h plasma glucose; HbA1c: glycosylated hemoglobin A1c; RCA: right coronary artery; LCA: left coronary artery; CAG: coronary angiography; TIMI: Thrombolysis in Myocardial Infarction Trial criteria; UCG: ultrasonic cardiogram.

\section{Acknowledgements}

This study was partly supported by grants from National Natural Science Foundation of China (No. 81270256). The authors would like to thank Shunping Zhou from Tongji University School of Medicine for her critical suggestion and Xiang Li from Tongji Law School/Intellectual Property Institution for providing information on medical insurance policies. We are also grateful to all participants in this study for their active cooperation.

\section{Competing Interests}

The authors have declared that no competing interest exists. 


\section{References}

1. O'Gara PT, Kushner FG, Ascheim DD, et al. American College of Emergency Physicians; Society for Cardiovascular Angiography and Interventions, 2013 ACCF/AHA Guideline for the management of ST-elevation myocardial infarction: a report of the American College of Cardiology Foundation/American Heart Association Task Force on Practice Guidelines. J Am Coll Cardiol. 2013; 61( e):78-140.

2. Yan $\mathrm{HB}$, Song $\mathrm{L}$, Chen $\mathrm{H}$, et al. Factors influencing ambulance use in patients with ST-elevation myocardial infarction in Beijing, China. Chin Med J (Engl). 2009; 122: 272-8.

3. Thuresson M, Jarlöv MB, Lindahl B, et al. Factors that influence the use of ambulance in acute coronary syndrome. Am Heart J. 2008; 156: 170-6.

4. Zhang SY, Hu DY, Sun YH, et al. Current management of patients with ST elevation myocardial infarction in Metropolitan Beijing, China. Clin Invest Med. 2008; 31: E189-97.

5. Wu WH, Yang L, Peng FH, et al. Lower socioeconomic status is associated with worse outcomes in pulmonary arterial hypertension. Am J Respir Crit Care Med. 2013; 187: 303-10.

6. Fang K, Shia B, Ma S. Health insurance coverage and impact: a survey in three cities in China. PLoS One. 2012; 7: e39157.

7. Tang L. The Chinese community patient's life satisfaction, assessment of community medical service, and trust in community health delivery system. Health Qual Life Outcomes. 2013; 11:18.

8. Kong Y, Wang Y, Zhang JH, et al. Disparities in medical expenditure and outcomes among patients with intracranial hemorrhage associated with different insurance statuses in southwestern China. Acta Neurochir Suppl. 2011; 111: 337-41.

9. Dong K. Medical insurance system evolution in China. China Econ Rev. 2009; 20: 591-7.

10. Nieminen MS, Böhm M, Cowie MR, et al. Executive summary of the guidelines on the diagnosis and treatment of acute heart failure: the Task Force on Acute Heart Failure of the European Society of Cardiology. Eur Heart J. 2005; 26: 384-416.

11. Brignole M, Occhetta E, Bongiorni MG, et al. Clinical evaluation of defibrillation testing in an unselected population of 2,120 consecutive patients undergoing first implantable cardioverter-defibrillator implant. J Am Coll Cardiol. 2012; 60: 981-7.

12. Ellis SG, Popma JJ, Lasala JM, et al. Relationship between angiographic late loss and target lesion revascularization after coronary stent implantation: analysis from the TAXUS-IV trial. J Am Coll Cardiol. 2005; 45: 1193-200.

13. Corpus RA, George PB, House JA, et al. Optimal glycemic control is associated with a lower rate of target vessel revascularization in treated type II diabetic patients undergoing elective percutaneous coronary intervention. J Am Coll Cardiol. 2004; 43: 8-14

14. Thygesen K, Alpert JS, Jaffe AS, et al. Third universal definition of myocardial infarction. J Am Coll Cardiol. 2012; 60:1581-98.

15. Mendis S, Thygesen K, Kuulasmaa K, et al. Writing group on behalf of the participating experts of the WHO consultation for revision of WHO definition of myocardial infarction. World Health Organization definition of myocardial infarction: 2008-09 revision. Int J Epidemiol. 2011; 40: 139-46.

16. Jauch EC, Saver JL, Adams HP Jr, et al. Guidelines for the early management of patients with acute ischemic stroke: a guideline for healthcare professionals from the American Heart Association/American Stroke Association. Stroke. 2013; 44: 870-947.

17. Sacco RL, Kasner SE, Broderick JP, et al. An updated definition of stroke for the 21st century: a statement for healthcare professionals from the American Heart Association/American Stroke Association. Stroke. 2013; 44: 2064-89.

18. Yancy CW, Jessup M, Bozkurt B, et al. 2013 ACCF/AHA guideline for the management of heart failure: a report of the American College of Cardiology Foundation/American Heart Association Task Force on Practice Guidelines. J Am Coll Cardiol. 2013; 62: e147-239.

19. Nowak D, Kozłowska H, Żurada A. The relationship between the dimensions of the right coronary artery and the type of coronary vasculature in human foetuses. Folia Morphol (Warsz). 2011; 70: 13-7.

20. Haase J, Escaned J, van Swijndregt EM, et al. Experimental validation of geometric and densitometric coronary measurements on the new generation Cardiovascular Angiography Analysis System (CAAS II). Cathet Cardiovasc Diagn. 1993; 30: 104-14

21. Morrow DA, Antman EM, Charlesworth A, et al. TIMI risk score for ST-elevation myocardial infarction: A convenient, bedside, clinical score for risk assessment at presentation: An intravenous nPA for treatment of infarcting myocardium early II trial substudy. Circulation. 2000; 102: 2031-7.

22. Brooks MM, Jones RH, Bach RG, et al. Predictors of mortality and mortality from cardiac causes in the bypass angioplasty revascularization investigation (BARI) randomized trial and registry. For the BARI Investigators. Circulation. 2000; 101: 2682-9.

23. Best PJ, Lennon $\mathrm{R}$, Ting $\mathrm{HH}$, et al. The impact of renal insufficiency on clinical outcomes in patients undergoing percutaneous coronary interventions. J Am Coll Cardiol. 2002; 39: 1113-9.

24. Guo R, Li Y, Xu Y, et al. Significance of fragmented QRS complexes for identifying culprit lesions in patients with non-ST-elevation myocardial infarction: a single-center, retrospective analysis of 183 cases. BMC Cardiovascular Disorders. 2012; 12: 44.
25. Tang S, Meng Q, Chen L, et al. Tackling the challenges to health equity in China. Lancet. 2008; 372: 1493-501.

26. Hesketh T, Ye XJ, Li L, et al. Health status and access to health care of migrant workers in China. Public Health Rep. 2008; 123:189-97.

27. Menees DS, Gurm HS. Door-to-balloon time and mortality. N Engl J Med. 2014; 370: 181-2.

28. Fang $\mathrm{P}$, Dong $\mathrm{S}$, Xiao J, et al. Regional inequality in health and its determinants: Evidence from China. Health Policy. 2010; 94: 14-25.

29. Hu S, Tang S, Liu Y, et al. Reform of how health care is paid for in China: challenges and opportunities. Lancet. 2008; 372:1846-53.

30. Liu Y, Berman P, Yip W, et al. Health care in China: the role of non-government providers. Health Policy. 2006; 77: 212-20.

31. Ying XH, Hu TW, Ren J, et al. Demand for private health insurance in Chinese urban areas. Health Econ. 2007; 16:1041-50.

32. Lin W, Liu GG, Chen G. The Urban Resident Basic Medical Insurance: a landmark reform towards universal coverage in China. Health Econ. 2009; 18: 83-96.

33. Zhao DH, Rao KQ, Zhang ZR. Coverage and utilization of the health insurance among migrant workers in Shanghai, China. Chin Med J (Engl). 2011; 12: 2328-34.

34. Li J, Zhang K, Tian L. Multiple facets of China's health inequality. Lancet. 2006; 367: 1397.

35. Bärnighausen $\mathrm{T}$, Liu $\mathrm{Y}$, Zhang $\mathrm{X}$, et al. Willingness to pay for social health insurance among informal sector workers in Wuhan, China: a contingent valuation study. BMC Health Serv Res. 2007; 7:114. 